\title{
Curved Nanotube Structures under Mechanical Loading
}

\author{
Hamidreza Yazdani Sarvestani and Ali Naghashpour \\ Concordia Centre for Composites (CONCOM), Department of Mechanical and Industrial Engineering, Concordia University, \\ Montreal, QC, Canada H3G 1M8 \\ Correspondence should be addressed to Hamidreza Yazdani Sarvestani; h_yazd@encs.concordia.ca
}

Received 25 April 2015; Accepted 2 July 2015

Academic Editor: Hassan Karimi-Maleh

Copyright @ $2015 \mathrm{H}$. Yazdani Sarvestani and A. Naghashpour. This is an open access article distributed under the Creative Commons Attribution License, which permits unrestricted use, distribution, and reproduction in any medium, provided the original work is properly cited.

\begin{abstract}
Configuration of carbon nanotube (CNT) has been the subject of research to perform theoretical development for analyzing nanocomposites. A new theoretical solution is developed to study curved nanotube structures subjected to mechanical loadings. A curved nanotube structure is considered. A nonlocal displacement-based solution is proposed by using a displacement approach of Toroidal Elasticity based on Eringen's theory of nonlocal continuum mechanics. The governing equations of curved nanotube structures are developed in toroidal coordinate system. The method of successive approximation is used to discretize the displacement-based governing equations and find the general solution subjected to bending moment. The numerical results show that all displacement components increase with increasing the nonlocal parameter. The present theoretical study highlights the significance of the geometry and nonlocal parameter effects on mechanical behavior of nanotube structures.
\end{abstract}

\section{Introduction}

Due to superior properties of CNT which offer unprecedented potential to produce multifunctional nanocomposites, many investigations have been performed on different applications of these nanostructures such as nanomechanical $[1,2]$ and nanobiological $[3,4]$ applications and optic [5] and molecular engineering [6-8]. CNTs appear in different shapes. Each shape has its own special properties and potential applications. CNT with curved configuration is one of their shapes. Actually, a single nanotube naturally curves during growth if no external forces exist [9]. Continuum mechanics approach has been employed by many researchers to study mechanical behavior of curved nanostructures.

The continuum mechanics approach has been used to investigate several aspects of mechanical analysis of CNT such as the static [10-13], the buckling [14-16], free vibration $[17,18]$, wave propagation [19-23], and thermomechanical analysis of CNT [24].

The effect of initial stress on the vibration of an individual multiwall carbon nanotube (MWCNT) with simply supported ends was investigated based on a laminated elastic beam model [12]. Wang et al. [14] proposed nonlocal elastic beam and shell models. They applied the proposed method to study the small-scale effect on buckling analysis of CNT under compression. Based on the continuum mechanics and the molecular dynamics simulation, the investigation on the wave propagation in a single-walled carbon nanotube (SWCNT) was performed [18]. Hu et al. [19] used nonlocal elastic beam theories to study the scale effect of the CNT on the wave dispersion. They compared the results obtained by nonlocal elastic Timoshenko beam theory with the results obtained by molecular dynamics simulations. Based on thermal elasticity mechanics and nonlocal elasticity theory, a single-elastic beam model was presented to investigate the thermal vibration of SWCNT [20]. Murmu and Pradhan [20] studied the buckling of SWCNT. They considered the effect of temperature change and surrounding elastic medium.

Compared with the classical continuum theory, the nonlocal elasticity theory uses the constitutive assumptions that the stress at a point is a function of strains at all points in the continuum [25-27]. Nonlocal elasticity theory was used by many researchers to analyze nanostructures advanced by Eringen [25, 26]. Peddieson et al. [27] used a version of nonlocal elasticity theory to propose a nonlocal Bernoulli-Euler beam model. Zhang et al. [28] presented a nonlocal double-elastic beam model for the free vibrations 
of double-walled carbon nanotubes (DWCNTs) based on theory of nonlocal elasticity and by considering the effect of small length scale. The elastic buckling analysis of nanotubes was analyzed based on Eringen's nonlocal elasticity Timoshenko beam theories $[29,30]$. The small-scale effect was considered through Eringen's nonlocal elasticity theory while the effect of transverse shear deformation was considered through Timoshenko beam theory. Frequency equations and modal shape functions of beam structures were derived based on a nonlocal Bernoulli-Euler beam [31].

The above review shows that little work has been done to study behavior of curved nanotube structures under mechanical loadings. A new displacement-based solution is proposed to study theoretically mechanical behavior of curved nanotube structures based on Toroidal Elasticity that includes the full three-dimensional constitutive relations. Governing equations of curved nanotube are developed by using the constitutive relations of Eringen. Then, the method of successive approximation is employed to find the general solution for mechanical behavior of curved nanotube structures subjected to pure bending moment. The effects of nonlocal parameter, nanotube length, and thickness on the mechanical behavior of nanotubes are studied.

\section{Nonlocal Theory (Constitutive Relations)}

According to Eringen $[25,26]$, the stress field at a point $x$ in an elastic continuum depends not only on the strain field at the point but also on strains at all other points of the body. Eringen attributed this fact to the atomic theory of lattice dynamics and experimental observations on phonon dispersion. Therefore, the following equivalent differential form is represented based on Eringen [25, 26]:

$$
\left(1-\mu \nabla^{2}\right) \sigma_{i j}=C_{i j m n} \varepsilon_{m n}
$$

where $\nabla^{2}$ and $\mu=\left(e_{0} a\right)^{2}$ are the Laplacian operator and nonlocal parameter, respectively. In addition, $e_{0}$ and $a$ are a material constant and internal characteristic length, respectively.

\section{Governing Equations for Curved Nanotube Structures}

The nonclassical toroidal coordinate system is shown in Figure 1. The curved nanotube has a bend radius $R$ and an annular cross section bounded by radii $a$ and $b$ (see Figure 1). A general point $P$ in a constant thickness curved nanotube is represented easily by the nonclassical toroidal coordinate system $r, \varphi$, and $\theta$, where $r$ and $\varphi$ are polar coordinates in the plane of the nanotube cross section and $\theta$ defines the position of the nanotube cross section. $\varsigma=$ $r / c$ is a nondimensional radial coordinate in the toroidal coordinate, and $c$ is a reference length to be specified later.

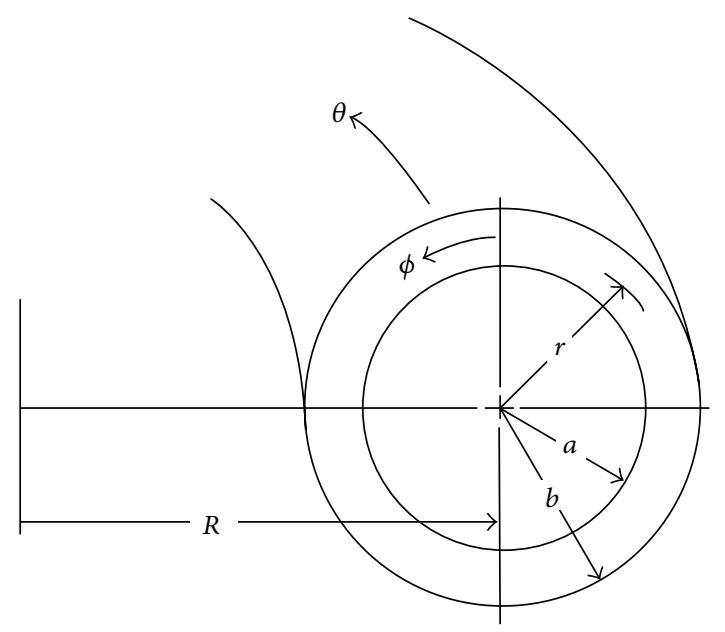

FIGURE 1: Geometry of curved nanotube and coordinate system.

For convenience, nondimensional displacements $u, v$, and $w$ are defined as

$$
\begin{gathered}
u=\frac{u_{\varsigma}}{c}, \\
v=\frac{u_{\phi}}{c}, \\
w=\frac{u_{\theta}}{c} .
\end{gathered}
$$

Nondimensional stress components are defined as

$$
\begin{gathered}
\tau_{\varsigma \varsigma}=\frac{1+v}{E} \sigma_{\varsigma \varsigma}, \\
\tau_{\phi \phi}=\frac{1+v}{E} \sigma_{\phi \phi}, \\
\tau_{\theta \theta}=\frac{1+v}{E} \sigma_{\theta \theta}, \\
\tau_{\varsigma \phi}=\frac{1+v}{E} \sigma_{\varsigma \phi}, \\
\tau_{\varsigma \theta}=\frac{1+v}{E} \sigma_{\varsigma \theta}, \\
\tau_{\phi \theta}=\frac{1+v}{E} \sigma_{\phi \theta},
\end{gathered}
$$

where $E$ and $v$ are modulus of elasticity and Poisson ratio for nanotubes. The kinematics relations for the case of small displacements are [34]

$$
\begin{aligned}
\varepsilon_{r} & =\frac{\partial u}{\partial r}, \\
\varepsilon_{\phi} & =\frac{u}{r}+\frac{1}{r} \frac{\partial v}{\partial \phi}, \\
\varepsilon_{\theta} & =\frac{1}{\rho}\left(u \cos \phi-v \sin \phi+\frac{\partial w}{\partial \theta}\right),
\end{aligned}
$$




$$
\begin{aligned}
\varepsilon_{r \phi} & =\frac{1}{2}\left(\frac{1}{r} \frac{\partial u}{\partial \phi}+\frac{\partial v}{\partial r}-\frac{v}{r}\right), \\
\varepsilon_{r \theta} & =\frac{1}{2}\left(\frac{\partial w}{\partial r}+\frac{1}{\rho} \frac{\partial u}{\partial \theta}-\frac{w}{\rho} \cos \phi\right), \\
\varepsilon_{\phi \theta} & =\frac{1}{2}\left(\frac{1}{r} \frac{\partial w}{\partial \phi}+\frac{1}{\rho} \frac{\partial v}{\partial \theta}+\frac{w}{\rho} \sin \phi\right),
\end{aligned}
$$

where $u, v$, and $w$ represent the displacement components in the $r, \varphi$, and $\theta$ directions, respectively. From both considerations of (1) and Hooke's law, it is written that

$$
\begin{gathered}
\left(\begin{array}{c}
\sigma_{\varsigma \varsigma} \\
\sigma_{\phi \phi} \\
\sigma_{\theta \theta} \\
\sigma_{\varsigma \phi} \\
\sigma_{\varsigma \theta} \\
\sigma_{\phi \theta}
\end{array}\right)-\mu \nabla^{2}\left(\begin{array}{c}
\sigma_{\varsigma \varsigma} \\
\sigma_{\phi \phi} \\
\sigma_{\theta \theta} \\
\sigma_{\zeta \phi} \\
\sigma_{\varsigma \theta} \\
\sigma_{\phi \theta}
\end{array}\right)=\frac{2}{(1+v)(1-2 v)} \\
\cdot\left[\begin{array}{cccccc}
1-v & v & v & 0 & 0 & 0 \\
v & 1-v & v & 0 & 0 & 0 \\
v & v & 1-v & 0 & 0 & 0 \\
0 & 0 & 0 & 1-2 v & 0 & 0 \\
0 & 0 & 0 & 0 & 1-2 v & 0 \\
0 & 0 & 0 & 0 & 0 & 1-2 v
\end{array}\right]\left(\begin{array}{c}
\varepsilon_{\varsigma \varsigma} \\
\varepsilon_{\phi \phi} \\
\varepsilon_{\theta \theta} \\
\gamma_{\zeta \phi} \\
\gamma_{\varsigma \theta} \\
\gamma_{\phi \theta}
\end{array}\right) .
\end{gathered}
$$

By considering (5), (3), and strain displacement relations (4), the nondimensional stress components may be expressed in terms of the three nondimensional displacement components and nonlocal parameter $\mu$ as

$$
\begin{aligned}
\tau_{\varsigma \varsigma} & =\frac{1}{\widehat{\mu}} \frac{\partial u}{\partial \varsigma}+\frac{1}{\widehat{\mu}} \frac{\nu}{1-2 \nu} \vartheta, \\
\tau_{\phi \phi} & =\frac{1}{\widehat{\mu}}\left(\frac{u}{\varsigma}+\frac{1}{\varsigma} \frac{\partial v}{\partial \phi}\right)+\frac{1}{\widehat{\mu}} \frac{\nu}{1-2 \nu} \vartheta, \\
\tau_{\theta \theta} & =\frac{1}{\widehat{\mu}} \frac{c}{\rho} \chi+\frac{1}{\widehat{\mu}} \frac{\nu}{1-2 \nu} \vartheta \\
\tau_{\varsigma \phi} & =\frac{1}{2 \widehat{\mu}}\left(\frac{1}{\varsigma} \frac{\partial u}{\partial \phi}+\frac{\partial v}{\partial \varsigma}-\frac{v}{\varsigma}\right), \\
\tau_{\varsigma \theta} & =\frac{1}{2 \widehat{\mu}}\left(\frac{\partial w}{\partial \varsigma}+\frac{c}{\rho} \frac{\partial u}{\partial \theta}-\frac{c}{\rho} w \cos \phi\right), \\
\tau_{\phi \theta} & =\frac{1}{2 \widehat{\mu}}\left(\frac{1}{\varsigma} \frac{\partial w}{\partial \phi}+\frac{c}{\rho} \frac{\partial v}{\partial \theta}+\frac{c}{\rho} w \sin \phi\right),
\end{aligned}
$$

where

$$
\begin{aligned}
& \vartheta=\psi+\frac{c}{\rho} \chi, \\
& \psi=\frac{\partial u}{\partial \varsigma}+\frac{u}{\varsigma}+\frac{1}{\varsigma} \frac{\partial v}{\partial \phi}, \\
& \chi=u \cos \phi-v \sin \phi+\frac{\partial w}{\partial \theta}, \\
& \widehat{\mu}=1+\frac{n^{2}}{\varsigma^{2}} \mu .
\end{aligned}
$$

Three equilibrium equations in stress form in the toroidal coordinate are represented as [34]

$$
\begin{aligned}
& \frac{\partial \tau_{\varsigma \varsigma}}{\partial \varsigma}+\frac{1}{\varsigma} \frac{\partial \tau_{\varsigma \varsigma}}{\partial \phi}+\frac{1}{\varsigma}\left(\tau_{\varsigma \varsigma}-\tau_{\phi \phi}\right) \\
& +\frac{c}{\rho}\left(\frac{\partial \tau_{\varsigma \theta}}{\partial \theta}+\left(\tau_{\varsigma \varsigma}-\tau_{\phi \phi}\right) \cos \phi-\tau_{\varsigma \phi} \sin \phi\right)=0, \\
& \frac{\partial \tau_{\varsigma \phi}}{\partial \varsigma}+\frac{1}{\varsigma} \frac{\partial \tau_{\phi \phi}}{\partial \phi}+\frac{2}{\varsigma} \tau_{\varsigma \phi} \\
& +\frac{c}{\rho}\left(\frac{\partial \tau_{\phi \theta}}{\partial \theta}+\tau_{\varsigma \phi} \cos \phi-\left(\tau_{\phi \phi}-\tau_{\theta \theta}\right) \sin \phi\right)=0, \\
& \frac{\partial \tau_{\varsigma \theta}}{\partial \varsigma}+\frac{1}{\varsigma} \frac{\partial \tau_{\phi \theta}}{\partial \phi}+\frac{1}{\varsigma} \tau_{\varsigma \theta} \\
& +\frac{c}{\rho}\left(\frac{\partial \tau_{\theta \theta}}{\partial \theta}+2 \tau_{\varsigma \theta} \cos \phi-2 \tau_{\phi \theta} \sin \phi\right)=0,
\end{aligned}
$$

where $\rho=R+r \cos \varphi$ is the radial cylindrical coordinate of the general point. Substituting the stress-displacement relations (6) into the equilibrium equations (8), the government Navier equations in toroidal coordinate for the curved nanotube are obtained as

$$
\begin{gathered}
U=-\left(\frac{c}{\rho}\right)^{1} \bar{U}-\left(\frac{c}{\rho}\right)^{2} \widehat{U}, \\
V=-\left(\frac{c}{\rho}\right)^{1} \bar{V}-\left(\frac{c}{\rho}\right)^{2} \widehat{V}, \\
W=-\left(\frac{c}{\rho}\right)^{1} \bar{W}-\left(\frac{c}{\rho}\right)^{2} \widehat{W},
\end{gathered}
$$

where

$$
\begin{aligned}
U= & \frac{1}{\widehat{\mu}} \nabla^{2} u-\frac{1}{\widehat{\mu}} \frac{u}{\varsigma^{2}}-\frac{2}{\varsigma^{2}} \frac{1}{\widehat{\mu}} \frac{\partial v}{\partial \phi}+\frac{1}{1-2 \nu} \frac{1}{\widehat{\mu}} \frac{\partial \psi}{\partial \varsigma}, \\
\bar{U}= & \cos \phi \frac{1}{\widehat{\mu}} \frac{\partial u}{\partial \varsigma}-\sin \phi \frac{1}{\widehat{\mu}}\left(\frac{1}{\varsigma} \frac{\partial u}{\partial \phi}-\frac{v}{\varsigma}\right) \\
& +\frac{1}{1-2 v} \frac{1}{\widehat{\mu}} \frac{\partial \chi}{\partial \varsigma},
\end{aligned}
$$




$$
\begin{aligned}
\widehat{U}= & \frac{1}{\widehat{\mu}} \frac{\partial^{2} u}{\partial \theta^{2}}-\cos \phi \frac{1}{\widehat{\mu}} \frac{\partial w}{\partial \theta}-\frac{2(1-v)}{1-2 v} \cos \phi \frac{1}{\widehat{\mu}} \chi, \\
V= & \frac{1}{\widehat{\mu}} \nabla^{2} v-\frac{1}{\widehat{\mu}} \frac{v}{\varsigma^{2}}+\frac{2}{\varsigma^{2}} \frac{1}{\widehat{\mu}} \frac{\partial u}{\partial \phi}+\frac{1}{1-2 v} \frac{1}{\varsigma} \frac{1}{\widehat{\mu}} \frac{\partial \psi}{\partial \phi}, \\
\bar{V}= & \cos \phi \frac{1}{\widehat{\mu}} \frac{\partial v}{\partial \varsigma}-\sin \phi \frac{1}{\widehat{\mu}}\left(\frac{1}{\varsigma} \frac{\partial v}{\partial \phi}+\frac{u}{\varsigma}\right) \\
& +\frac{1}{1-2 v} \frac{1}{\varsigma} \frac{1}{\widehat{\mu}} \frac{\partial \chi}{\partial \phi}, \\
\widehat{V}= & \frac{1}{\widehat{\mu}} \frac{\partial^{2} v}{\partial \theta^{2}}+\sin \phi \frac{1}{\widehat{\hat{\mu}}} \frac{\partial w}{\partial \theta}+\frac{2(1-v)}{1-2 v} \sin \phi \frac{1}{\widehat{\mu}} \chi, \\
W= & \frac{1}{\widehat{\mu}} \nabla^{2} w, \\
\bar{W}= & \cos \phi \frac{1}{\widehat{\mu}} \frac{\partial w}{\partial \varsigma}-\sin \phi \frac{1}{\varsigma} \frac{1}{\widehat{\mu}} \frac{\partial w}{\partial \phi}+\frac{1}{1-2 v} \frac{1}{\widehat{\mu}} \frac{\partial \psi}{\partial \theta}, \\
\widehat{W}= & -\frac{1}{\widehat{\mu}} \frac{\partial^{2} w}{\partial \theta^{2}}-\frac{1}{\hat{\mu}} w+\frac{3-4 v}{1-2 v} \frac{1}{\widehat{\mu}} \frac{\partial \chi}{\partial \theta}, \\
\nabla^{2}(\ldots)= & \frac{\partial^{2}(\ldots)}{\partial \varsigma^{2}}+\frac{1}{\varsigma} \frac{\partial(\ldots)}{\partial \varsigma}+\frac{1}{\varsigma^{2}} \frac{\partial^{2}(\ldots)}{\partial \phi^{2}} .
\end{aligned}
$$

The three Navier equations serve as the fundamental equations for the curved nanotube. These equations are composed of three parts. The first part is independent of $c / \rho$. The second and the third parts are the linear and nonlinear parts of $c / \rho$. As it is impossible to find an exact solution for the Navier equations, the method of successive approximation is used to obtain an approximate solution.

\section{Method of Successive Approximation}

The method of successive approximation is employed to solve the Navier equations in the toroidal coordinate system. The solution for each component of the displacement is assumed to be a series in terms of a small parameter $\varepsilon$. The parameter $\varepsilon$ is chosen as

$$
\varepsilon=\frac{c}{R}
$$

Therefore the nondimensional displacement components take the form as

$$
\begin{gathered}
u=u_{0}+\varepsilon u_{1}+\varepsilon^{2} u_{2}+\varepsilon^{3} u_{3}+\cdots, \\
v=v_{0}+\varepsilon v_{1}+\varepsilon^{2} v_{2}+\varepsilon^{3} v_{3}+\cdots, \\
w=w_{0}+\varepsilon w_{1}+\varepsilon^{2} w_{2}+\varepsilon^{3} w_{3}+\cdots,
\end{gathered}
$$

where the indexes of the right hand side terms indicate the order of the displacement components in the series. By substituting the series form displacement functions (12) into
(3), the corresponding nondimensional stress components are derived as

$$
\begin{aligned}
& \tau_{\varsigma \varsigma}=\tau_{\varsigma \varsigma 0}+\varepsilon \tau_{\varsigma \varsigma 1}+\varepsilon^{2} \tau_{\varsigma \varsigma 2}+\varepsilon^{3} \tau_{\varsigma \varsigma 3}+\cdots, \\
& \tau_{\phi \phi}=\tau_{\phi \phi 0}+\varepsilon \tau_{\phi \phi 1}+\varepsilon^{2} \tau_{\phi \phi 2}+\varepsilon^{3} \tau_{\phi \phi 3}+\cdots \\
& \tau_{\theta \theta}=\tau_{\theta \theta 0}+\varepsilon \tau_{\theta \theta 1}+\varepsilon^{2} \tau_{\theta \theta 2}+\varepsilon^{3} \tau_{\theta \theta 3}+\cdots, \\
& \tau_{\varsigma \phi}=\tau_{\varsigma \phi 0}+\varepsilon \tau_{\varsigma \phi 1}+\varepsilon^{2} \tau_{\varsigma \phi 2}+\varepsilon^{3} \tau_{\varsigma \phi 3}+\cdots \\
& \tau_{\varsigma \theta}=\tau_{\varsigma \theta 0}+\varepsilon \tau_{\varsigma \theta 1}+\varepsilon^{2} \tau_{\varsigma \theta 2}+\varepsilon^{3} \tau_{\varsigma \theta 3}+\cdots \\
& \tau_{\phi \theta}=\tau_{\phi \theta 0}+\varepsilon \tau_{\phi \theta 1}+\varepsilon^{2} \tau_{\phi \theta 2}+\varepsilon^{3} \tau_{\phi \theta 3}+\cdots
\end{aligned}
$$

The functions of Navier equations are expanded as

$$
\begin{aligned}
& U=U_{0}+\varepsilon U_{1}+\varepsilon^{2} U_{2}+\varepsilon^{3} U_{3}+\cdots \\
& \bar{U}=\bar{U}_{0}+\varepsilon \bar{U}_{1}+\varepsilon^{2} \bar{U}_{2}+\varepsilon^{3} \bar{U}_{3}+\cdots \\
& \widehat{U}=\widehat{U}_{0}+\varepsilon \widehat{U}_{1}+\varepsilon^{2} \widehat{U}_{2}+\varepsilon^{3} \widehat{U}_{3}+\cdots \\
& V=V_{0}+\varepsilon V_{1}+\varepsilon^{2} V_{2}+\varepsilon^{3} V_{3}+\cdots \\
& \bar{V}=\bar{V}_{0}+\varepsilon \bar{V}_{1}+\varepsilon^{2} \bar{V}_{2}+\varepsilon^{3} \bar{V}_{3}+\cdots \\
& \widehat{V}=\widehat{V}_{0}+\varepsilon \widehat{V}_{1}+\varepsilon^{2} \widehat{V}_{2}+\varepsilon^{3} \widehat{V}_{3}+\cdots \\
& W=W_{0}+\varepsilon W_{1}+\varepsilon^{2} W_{2}+\varepsilon^{3} W_{3}+\cdots \\
& \bar{W}=\bar{W}_{0}+\varepsilon \bar{W}_{1}+\varepsilon^{2} \bar{W}_{2}+\varepsilon^{3} \bar{W}_{3}+\cdots \\
& \widehat{W}=\widehat{W}_{0}+\varepsilon \widehat{W}_{1}+\varepsilon^{2} \widehat{W}_{2}+\varepsilon^{3} \widehat{W}_{3}+\cdots
\end{aligned}
$$

By substituting the expanded Navier functions (14) into Navier equations (9), matching terms in $\varepsilon$, and setting each order of $\varepsilon$ to zero, the governing equations for the different orders are obtained. The details to obtain the solution are explained in Appendix A.

\section{General Solution for Curved Nanotubes Subjected to Pure Bending}

A direct solution is formulated to avoid the complications that arise from the use of stress function. The complementary solution for the displacement is given in the following form:

$$
\begin{aligned}
& u_{0}=a \varsigma^{m} \cos (n \phi) \cos (\theta), \\
& v_{0}=b \varsigma^{m} \sin (n \phi) \cos (\theta), \\
& w_{0}=c \varsigma^{\bar{m}} \cos (n \phi) \sin (\theta) .
\end{aligned}
$$




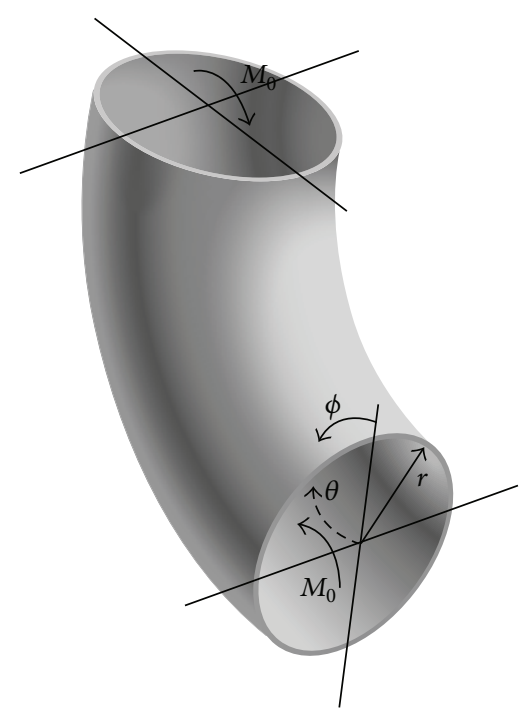

Figure 2: End couples acting on the curved nanotube.

The quantities $a, b, c, m, \bar{m}$, and $n$ are constants determined from zeroth-order Navier equations (13) and stress free boundary conditions on the free surfaces. The results are

$$
\begin{aligned}
u_{0} & =\left[a_{0} \varsigma^{-1}+b_{0} \varsigma+\left\{a_{1}+b_{1}(1-4 \nu) \varsigma^{2}+c_{1} \varsigma^{-2}\right.\right. \\
& \left.+d_{1}(3-4 \nu) \ln \varsigma\right\} \cos \phi+\sum_{n=2}^{\infty}\left\{a_{n} \varsigma^{n-1}\right. \\
& +b_{n}(2-n-4 \nu) \varsigma^{n+1}+c_{n} \varsigma^{-(n+1)} \\
& \left.\left.+d_{n}(2-n+4 \nu) \varsigma^{-(n-1)}\right\} \cos n \phi\right] \cos (\theta), \\
v_{0} & =\left[\left\{-a_{1}+b_{1}(5-4 \nu) \varsigma^{2}+c_{1} \varsigma^{-2}-d_{1}(3-4 \nu) \ln \varsigma\right.\right. \\
& \left.-d_{1}\right\} \sin \phi+\sum_{n=2}^{\infty}\left\{-a_{n} \varsigma^{n-1}+b_{n}(4+n-4 \nu) \varsigma^{n+1}\right. \\
& \left.\left.+c_{n} \varsigma^{-(n+1)}+d_{n}(4-n-4 \nu) \varsigma^{-(n-1)}\right\} \sin n \phi\right] \cos (\theta), \\
& \left.\left.+f_{n} \varsigma^{n}\right\} \cos n \phi\right] \sin (\theta), \\
w_{0} & =\left[e_{0}+f_{0} \ln \varsigma+\left\{e_{1} \varsigma^{-1}+f_{1} \varsigma\right\} \cos \phi+\sum_{n=2}^{\infty}\left\{e_{n} \varsigma^{-n}\right.\right.
\end{aligned}
$$

where the quantities $a_{0}, b_{0}, a_{1}, b_{1}, c_{1}, d_{1}, e_{0}, f_{0}, e_{1}, f_{1}, a_{n}, b_{n}$, $c_{n}, d_{n}, e_{n}$, and $f_{n}$ are constants to be found from the boundary conditions. The detailed solution is presented for the curved nanotube subjected to bending moments, $M_{0}$, as shown in Figure 2.

Solutions are proposed successively for the various orders, starting from the zeroth order. The zeroth-order solution is taken from the complementary solution. The particular part is arranged using trial functions. The displacement functions for that order are obtained by summing the complementary and particular parts. The constants in the complementary solution of each order are determined separately from the boundary conditions. The total solution is the sum of the particular and complementary solution of all the orders times the appropriate power of $\varepsilon$. Any constants remaining in the total solution are determined from end or edge boundary conditions.

5.1. The Zeroth-Order Solution. Considering the boundary conditions on the inner and outer free surfaces, the displacement components are obtained as

$$
\begin{aligned}
& u_{0}=a_{1} \cos \phi \cos (\theta), \\
& v_{0}=-a_{1} \sin \phi \cos (\theta), \\
& w_{0}=0 .
\end{aligned}
$$

Constant $a_{1}$ of this order is to be determined later from the boundary conditions on the ends of the curved nanotube structure.

5.2. The First-Order Solution. The first-order displacement is obtained as

$$
\begin{gathered}
u_{1}=0, \\
v_{1}=0, \\
w_{1}=0 .
\end{gathered}
$$

5.3. The Second-Order Solution. The second-order displacement is obtained as

$$
\begin{aligned}
& u_{2}=A_{1} \varsigma^{2} \cos \phi \cos (\theta), \\
& v_{2}=B_{1} \varsigma^{2} \sin \phi \cos (\theta), \\
& w_{2}=0,
\end{aligned}
$$

where

$$
\begin{aligned}
& A_{1}=\frac{1}{\widehat{\mu}} \frac{4 v^{2}-13 v+4}{4 v-2} a_{1}, \\
& B_{1}=\frac{1}{\widehat{\mu}} \frac{4 v^{2}-9 v+8}{4 v-2} a_{1} .
\end{aligned}
$$

5.4. The Third-Order Solution. The third-order solution then takes the form

$$
\begin{aligned}
u_{3} & =\left\{a_{2} \varsigma+b_{2}(1-4 \nu) \varsigma^{3}+c_{2} \varsigma^{-3}+d_{2}(3-4 \nu) \varsigma^{-1}\right. \\
& \left.+A_{22} \varsigma^{3}\right\} \cos 2 \phi \cos (\theta), \\
v_{3} & =\left\{-a_{2} \varsigma+b_{2}(6-4 \nu) \varsigma^{3}+c_{2} \varsigma^{-3}+d_{2}(2-4 \nu) \varsigma^{-1}\right. \\
& \left.+C_{22} \varsigma^{3}\right\} \sin 2 \phi \cos (\theta), \\
w_{3} & =\left\{e_{2} \varsigma^{-2}+f_{2} \varsigma^{2}+D_{22} \varsigma^{3}\right\} \cos \phi \sin (\theta),
\end{aligned}
$$


where $a_{2}, b_{2}, c_{2}, d_{2}, e_{2}$, and $f_{2}$ are free constants. These are determined from the boundary conditions and governing equations. These constants are functions of the material property, $v$, the ratio $\lambda=a / b$, and the nonlocal parameter $\mu$. The coefficients appearing in (21) are defined in Appendix B.

5.5. The Fourth-Order Solution. The fourth-order solution takes the form

$$
\begin{aligned}
u_{4} & =\left[a_{3} \varsigma^{2}-b_{3}(1+4 v) \varsigma^{4}+c_{3} \varsigma^{-4}-d_{3}(5-4 \nu) \varsigma^{-2}\right] \\
& \cdot \cos 3 \phi \cos (\theta)+\frac{1-2 v}{20 v-11}\left[L_{1} \varsigma^{4}+L_{2} \varsigma^{-2}+L_{3}\right] \\
& \cdot \cos 3 \phi \cos (\theta)+\frac{1-2 v}{4 v-3}\left[L_{4} \varsigma^{2}+L_{5} \varsigma^{4}+L_{6}\right] \cos \phi \\
& \cdot \cos (\theta), \\
v_{4} & =\left[-a_{3} \varsigma^{2}+b_{3}(7-4 \nu) \varsigma^{4}+c_{3} \varsigma^{-4}+d_{3}(1-4 \nu) \varsigma^{-2}\right] \\
& \cdot \sin 3 \phi \cos (\theta)+\frac{1-2 v}{20 v-19}\left[L_{7} \varsigma^{4}+L_{8} \varsigma^{-2}+L_{9}\right] \\
& \cdot \sin 3 \phi \cos (\theta) \\
& +\frac{1-2 v}{4 v-3}\left[L_{10} \varsigma^{2}+L_{11} \varsigma^{4}+L_{12}+L_{13} \varsigma^{-1}+L_{14} \varsigma^{13}\right] \\
& \cdot \sin \phi \cos (\theta), \\
w_{4} & =\left[e_{3} \varsigma^{-3}+f_{3} \varsigma^{3}\right] \cos 2 \phi \sin (\theta) \\
& +\frac{-1}{4}\left[L_{15} \varsigma^{-1}+L_{16} \varsigma^{3}+L_{18}+L_{17} \varsigma^{4}\right] \cos 2 \phi \\
& \cdot \sin (\theta) .
\end{aligned}
$$

It should be noted that $a_{3}, b_{3}, c_{3}, d_{3}, e_{3}$, and $f_{3}$ are free constants obtained in Appendix $\mathrm{C}$ by applying the boundary conditions and governing equations. Finally, substituting the solution for the various orders into (12) yields the total solution for the problem.

\section{Results and Discussion}

The theoretical investigations are performed for the curved single-walled carbon nanotube (SWCNT) subjected to bending moment (see Figure 2). The mechanical properties of SWCNT are given in Table 1 [32]. Furthermore, the displacement components are normalized as $\bar{u}_{i}=u_{i} / u_{0}$, where $u_{0}=$ $L * \sigma_{0} / E$ and $\sigma_{0}=\left(M_{0} \cdot r\right) /(\pi / 64) *\left(O D^{4}-I D^{4}\right)$ where $O D$ and $I D$ are outer and inner radius of the curved SWCNT. The innermost radius and thickness of SWCNT are assumed to be $8.5 \mathrm{~nm}$ and $0.34 \mathrm{~nm}$, respectively [33]. In addition, $L$ is the length of the curved nanotube. $L / h$ is defined as an aspect ratio.

Tube length, thickness, bend radius, and nonlocal parameter have effects on the nondimensional displacement components. The numerical results for curved SWCNT under bending moment $\left(M_{0}\right)$ at the point in which $\phi=180^{\circ}$ are presented in Tables 2, 3, 4, and 5 .
TABLE 1: Mechanical properties of SWCNT [32].

\begin{tabular}{lcc}
\hline \multirow{2}{*}{ Nanotubes } & \multicolumn{2}{c}{ Mechanical properties } \\
& $E(\mathrm{TPa})$ & $v$ (Poisson ratio) \\
\hline SWCNT & 1.05 & 0.19 \\
\hline
\end{tabular}

TABLE 2: Nondimensional displacement components of curved $\operatorname{SWCNT}(R / D=4$ and $L=r * \pi / 2)$.

\begin{tabular}{ccccc}
\hline$L / h$ & $\mu$ & $\bar{u}_{\theta}$ & $\bar{u}_{\phi}\left(\times 10^{-2}\right)$ & $\bar{u}_{r}\left(\times 10^{-4}\right)$ \\
\hline \multirow{4}{*}{100} & 0 & 1.158 & 1.390 & 1.454 \\
& 1.0 & 2.045 & 2.356 & 1.998 \\
& 2.0 & 2.081 & 2.505 & 2.455 \\
& 3.0 & 2.305 & 2.890 & 2.531 \\
& 4.0 & 2.712 & 3.104 & 3.104 \\
& 5.0 & 3.019 & 3.505 & 3.402 \\
\hline \multirow{4}{*}{200} & 0 & 1.401 & 1.682 & 1.759 \\
& 1.0 & 2.474 & 2.851 & 2.418 \\
& 2.0 & 2.518 & 3.031 & 2.971 \\
& 3.0 & 2.789 & 3.497 & 3.063 \\
& 4.0 & 3.282 & 3.756 & 3.756 \\
& 5.0 & 3.653 & 4.241 & 4.116 \\
\hline \multirow{4}{*}{500} & 0 & 1.760 & 2.113 & 2.210 \\
& 1.0 & 3.108 & 3.581 & 3.037 \\
& 2.0 & 3.163 & 3.808 & 3.732 \\
& 3.0 & 3.504 & 4.393 & 3.847 \\
& 4.0 & 4.122 & 4.718 & 4.718 \\
& 5.0 & 4.589 & 5.328 & 5.171 \\
\hline
\end{tabular}

TABLE 3: Nondimensional displacement components of curved $\operatorname{SWCNT}(R / D=4$ and $L=r * \pi)$.

\begin{tabular}{ccccc}
\hline$L / h$ & $\mu$ & $\bar{u}_{\theta}$ & $\bar{u}_{\phi}\left(\times 10^{-2}\right)$ & $\bar{u}_{r}\left(\times 10^{-4}\right)$ \\
\hline \multirow{4}{*}{100} & 0 & 1.285 & 1.543 & 1.614 \\
& 1.0 & 2.270 & 2.615 & 2.218 \\
& 2.0 & 2.310 & 2.781 & 2.725 \\
& 3.0 & 2.559 & 3.208 & 2.809 \\
& 4.0 & 3.010 & 3.445 & 3.445 \\
& 5.0 & 3.351 & 3.891 & 3.776 \\
\hline \multirow{4}{*}{200} & 0 & 1.581 & 1.898 & 1.985 \\
& 1.0 & 2.792 & 3.216 & 2.728 \\
& 2.0 & 2.841 & 3.421 & 3.352 \\
& 3.0 & 3.148 & 3.946 & 3.455 \\
& 4.0 & 3.702 & 4.237 & 4.237 \\
& 5.0 & 4.122 & 4.786 & 4.644 \\
\hline \multirow{4}{*}{500} & 0 & 1.979 & 2.376 & 2.486 \\
& 1.0 & 3.496 & 4.027 & 3.416 \\
& 2.0 & 3.557 & 4.283 & 4.197 \\
& 3.0 & 3.941 & 4.940 & 4.326 \\
& 4.0 & 4.635 & 5.305 & 5.305 \\
& 5.0 & 5.161 & 5.992 & 5.815 \\
\hline
\end{tabular}

The effects of thickness and nonlocal parameter $(\mu)$ on the nondimensional displacement components are investigated while assuming $R / O D=4$ and $L=r * \pi / 2$ as shown 
TABLE 4: Nondimensional displacement components of curved $\operatorname{SWCNT}(\mu=3.0$ and $L=r * \pi / 2)$.

\begin{tabular}{ccccc}
\hline$L / h$ & $R / D$ & $\bar{u}_{\theta}$ & $\bar{u}_{\phi}\left(\times 10^{-2}\right)$ & $\bar{u}_{r}\left(\times 10^{-4}\right)$ \\
\hline \multirow{3}{*}{100} & 4 & 2.305 & 2.890 & 2.531 \\
& 6 & 2.265 & 2.701 & 2.508 \\
& 8 & 2.201 & 2.692 & 2.420 \\
& 10 & 2.165 & 2.669 & 2.398 \\
\hline \multirow{4}{*}{200} & 4 & 2.881 & 3.613 & 3.164 \\
& 6 & 2.831 & 3.376 & 3.135 \\
& 8 & 2.751 & 3.365 & 3.025 \\
& 10 & 2.706 & 3.336 & 2.998 \\
\hline \multirow{3}{*}{500} & 4 & 3.596 & 4.508 & 3.948 \\
& 6 & 3.533 & 4.214 & 3.912 \\
& 8 & 3.434 & 4.200 & 3.775 \\
& 10 & 3.377 & 4.164 & 3.741 \\
\hline
\end{tabular}

TABLE 5: Nondimensional displacement components of curved SWCNT $(\mu=3.0$ and $L=r * \pi)$.

\begin{tabular}{ccccc}
\hline$L / h$ & $R / D$ & $\bar{u}_{\theta}$ & $\bar{u}_{\phi}\left(\times 10^{-2}\right)$ & $\bar{u}_{r}\left(\times 10^{-4}\right)$ \\
\hline \multirow{3}{*}{100} & 4 & 2.582 & 3.237 & 2.835 \\
& 6 & 2.537 & 3.025 & 2.809 \\
& 8 & 2.465 & 3.015 & 2.710 \\
& 10 & 2.425 & 2.989 & 2.686 \\
\hline \multirow{3}{*}{200} & 4 & 3.202 & 4.014 & 3.515 \\
& 6 & 3.146 & 3.751 & 3.483 \\
& 8 & 3.057 & 3.739 & 3.360 \\
& 10 & 3.007 & 3.706 & 3.331 \\
\hline \multirow{3}{*}{500} & 4 & 4.002 & 5.017 & 4.394 \\
& 6 & 3.932 & 4.689 & 4.354 \\
& 8 & 3.821 & 4.673 & 4.201 \\
& 10 & 3.759 & 4.633 & 4.163 \\
\hline
\end{tabular}

in Table 2. It is found from Table 2 that the displacement components increase with increasing the aspect ratio $(L / h)$ while curved SWCNT length is kept constant. The displacement components increase with the increase of the nonlocal parameter $(\mu)$ while the length and thickness of curved SWCNT are kept constant as shown in Table 2.

Table 3 shows the effects of thickness and nonlocal parameter $(\mu)$ on the nondimensional displacement components for $R / O D=4$ and $L=r * \pi$. It is observed from Table 3 that the displacement components increase with increasing the aspect ratio $(L / h)$ while curved SWCNT length is kept constant. Observing Table 3, the displacement components increase as the nonlocal parameter $(\mu)$ increases where length and thickness of curved SWCNT are fixed.

Comparing Table 2 with Table 3 , it is clear that the nondimensional displacement components increase with increasing the curved SWCNT length.

The effects of thickness and bend radius ratio $(R / D)$ on the nondimensional displacement components are studied for $\mu=3.0$ and $L=r * \pi / 2$ as shown in Table 4 . Observing Table 4, the displacement components decrease with the increase of bend radius ratio $(R / D)$.
Table 5 shows the effects of thickness and bend radius ratio $(R / D)$ on the nondimensional displacement components for $\mu=3.0$ and $L=r * \pi / 2$. It is observed from Table 5 that the displacement components increase with increasing the aspect ratio $(L / h)$ while curved SWCNT length is kept constant. Table 5 shows that the displacement components decrease as the bend radius ratio $(R / D)$ increases where length and thickness of curved SWCNT are fixed.

\section{Conclusions}

We have presented a new displacement-based solution by using the nonlocal Eringen theory and Toroidal Elasticity to study curved nanotube structures under mechanical loadings. Equations of motion were developed based on Eringen's differential constitutive equations of nonlocal elasticity for curved nanotube structures. The successive approximation method was employed to simplify the governing equations for curved nanotube structures under pure bending moment. The numerical results demonstrate the effectiveness of length, thickness, bend radius, and nonlocal parameter on all displacement components of curved nanotubes. It was observed that for curved SWCNT the values of displacement component in the longitudinal direction $\left(\bar{u}_{\theta}\right)$ were greater than those for the displacement components in the circumferential $\left(\bar{u}_{\phi}\right)$ and radial $\left(\bar{u}_{r}\right)$ directions.

\section{Appendices}

\section{A. Governing Equations for the Different Orders}

The equations are expressed for the zeroth order as

$$
\begin{gathered}
U_{0}=0 ; \\
V_{0}=0 ; \\
W_{0}=0 .
\end{gathered}
$$

For the first order

$$
\begin{aligned}
U_{1} & =-\bar{U}_{0} ; \\
V_{1} & =-\bar{V}_{0} ; \\
W_{1} & =-\bar{W}_{0} .
\end{aligned}
$$

For the second order

$$
\begin{gathered}
U_{2}=-\bar{U}_{1}+\bar{U}_{0} \varsigma \cos \phi-\widehat{U}_{0}, \\
V_{2}=-\bar{V}_{1}+\bar{V}_{0} \varsigma \cos \phi-\widehat{V}_{0}, \\
W_{2}=-\bar{W}_{1}+\bar{W}_{0} \varsigma \cos \phi-\widehat{W}_{0} .
\end{gathered}
$$


For the third order

$$
\begin{aligned}
U_{3}= & -\bar{U}_{2}+\bar{U}_{1} \varsigma \cos \phi-\bar{U}_{0} \varsigma^{2} \cos ^{2} \phi-\widehat{U}_{1} \\
& +2 \widehat{U}_{0} \varsigma \cos \phi, \\
V_{3}= & -\bar{V}_{2}+\bar{V}_{1} \varsigma \cos \phi-\bar{V}_{0} \varsigma^{2} \cos ^{2} \phi-\widehat{V}_{1} \\
& +2 \widehat{V}_{0} \varsigma \cos \phi, \\
W_{3}= & -\bar{W}_{2}+\bar{W}_{1} \varsigma \cos \phi-\bar{W}_{0} \varsigma^{2} \cos ^{2} \phi-\widehat{W}_{1} \\
& +2 \widehat{W}_{0} \varsigma \cos \phi .
\end{aligned}
$$

And for the fourth order

$$
\begin{aligned}
U_{4}= & -\bar{U}_{3}+\bar{U}_{2} \varsigma \cos \phi-\bar{U}_{1} \varsigma^{2} \cos ^{2} \phi+\bar{U}_{0} \varsigma^{3} \cos ^{3} \phi \\
& -\widehat{U}_{2}+2 \widehat{U}_{1} \varsigma \cos \phi-3 \widehat{U}_{0} \varsigma^{2} \cos ^{2} \phi \\
V_{4}= & -\bar{V}_{3}+\bar{V}_{2} \varsigma \cos \phi-\bar{V}_{1} \varsigma^{2} \cos ^{2} \phi+\bar{V}_{0} \varsigma^{3} \cos ^{3} \phi \\
& -\widehat{V}_{2}+2 \widehat{V}_{1} \varsigma \cos \phi-3 \widehat{V}_{0} \varsigma^{2} \cos ^{2} \phi \\
W_{4}= & -\bar{W}_{3}+\bar{W}_{2} \varsigma \cos \phi-\bar{W}_{1} \varsigma^{2} \cos ^{2} \phi+\bar{W}_{0} \varsigma^{3} \cos ^{3} \phi \\
& -\widehat{W}_{2}+2 \widehat{W}_{1} \varsigma \cos \phi-3 \widehat{W}_{0} \varsigma^{2} \cos ^{2} \phi .
\end{aligned}
$$

The Navier functions of order $k(k=0,1,2, \ldots)$ in (A.1)(A.5) are defined as

$$
\begin{aligned}
& U_{k}=\frac{1}{\widehat{\mu}} \nabla^{2} u_{k}-\frac{1}{\widehat{\mu}} \frac{u_{k}}{\varsigma^{2}}-\frac{2}{\varsigma^{2}} \frac{1}{\widehat{\mu}} \frac{\partial v_{k}}{\partial \phi} \\
& +\frac{1}{1-2 \nu} \frac{1}{\widehat{\mu}} \frac{\partial \psi_{k}}{\partial \varsigma} \\
& \bar{U}_{k}=\cos \phi \frac{1}{\widehat{\mu}} \frac{\partial u_{k}}{\partial \varsigma}-\sin \phi \frac{1}{\widehat{\mu}}\left(\frac{1}{\varsigma} \frac{\partial u_{k}}{\partial \phi}-\frac{v_{k}}{\varsigma}\right) \\
& +\frac{1}{1-2 v} \frac{1}{\widehat{\mu}} \frac{\partial \chi_{k}}{\partial \varsigma} \\
& \widehat{U}_{k}=\frac{1}{\widehat{\mu}} \frac{\partial^{2} u_{k}}{\partial \theta^{2}}-\cos \phi \frac{1}{\widehat{\mu}} \frac{\partial w_{k}}{\partial \theta} \\
& -\frac{2(1-v)}{1-2 v} \cos \phi \frac{1}{\widehat{\mu}} \chi_{k}, \\
& V_{k}=\frac{1}{\widehat{\mu}} \nabla^{2} v_{k}-\frac{1}{\widehat{\mu}} \frac{v_{k}}{\varsigma^{2}}+\frac{2}{\varsigma^{2}} \frac{1}{\widehat{\mu}} \frac{\partial u_{k}}{\partial \phi} \\
& +\frac{1}{1-2 \nu} \frac{1}{\varsigma} \frac{1}{\widehat{\mu}} \frac{\partial \psi_{k}}{\partial \phi} \\
& \bar{V}_{k}=\cos \phi \frac{1}{\widehat{\mu}} \frac{\partial v_{k}}{\partial \varsigma}-\sin \phi \frac{1}{\widehat{\mu}}\left(\frac{1}{\varsigma} \frac{\partial v_{k}}{\partial \phi}+\frac{u_{k}}{\varsigma}\right) \\
& +\frac{1}{1-2 v} \frac{1}{\varsigma} \frac{1}{\widehat{\mu}} \frac{\partial \chi_{k}}{\partial \phi},
\end{aligned}
$$

$$
\begin{aligned}
\widehat{V}_{k}= & \frac{1}{\widehat{\mu}} \frac{\partial^{2} v_{k}}{\partial \theta^{2}}+\sin \phi \frac{1}{\widehat{\mu}} \frac{\partial w_{k}}{\partial \theta} \\
& +\frac{2(1-v)}{1-2 v} \sin \phi \frac{1}{\widehat{\mu}} \chi_{k}, \\
W_{k}= & \frac{1}{\widehat{\mu}} \nabla^{2} w_{k}, \\
\bar{W}_{k}= & \cos \phi \frac{1}{\widehat{\mu}} \frac{\partial w_{k}}{\partial \varsigma}-\sin \phi \frac{1}{\varsigma} \frac{1}{\widehat{\mu}} \frac{\partial w_{k}}{\partial \phi} \\
& +\frac{1}{1-2 v} \frac{1}{\widehat{\mu}} \frac{\partial \psi_{k}}{\partial \theta}, \\
\widehat{W}_{k}= & -\frac{1}{\widehat{\mu}} \frac{\partial^{2} w_{k}}{\partial \theta^{2}}-\frac{1}{\widehat{\mu}} w_{k}+\frac{3-4 v}{1-2 v} \frac{1}{\widehat{\mu}} \frac{\partial \chi_{k}}{\partial \theta} \\
\psi_{k}= & \frac{1}{\widehat{\mu}} \frac{\partial u_{k}}{\partial \varsigma}+\frac{1}{\widehat{\mu}} \frac{u_{k}}{\varsigma}+\frac{1}{\varsigma} \frac{1}{\widehat{\mu}} \frac{\partial v_{k}}{\partial \phi}, \\
\nabla_{k}^{2}(\ldots)= & \frac{1}{\widehat{\mu}} u_{k} \cos \phi-\frac{1}{\widehat{\mu}} v_{k} \sin \phi+\frac{1}{\widehat{\mu}} \frac{\partial w_{k}}{\partial \theta} \\
& \frac{\partial^{2}}{\partial \varsigma^{2}}+\frac{1}{\varsigma} \frac{\partial(\ldots)}{\partial \varsigma}+\frac{1}{\varsigma^{2}} \frac{\partial^{2}(\ldots)}{\partial \phi^{2}}
\end{aligned}
$$

The displacement functions on the right hand members with negative indexes are assumed to be zero. It is noted from (A.1)-(A.5) that the zeroth-order governing equations are homogenous equations. The right hand side terms for orders greater than zero contain portions from the lower orders, and the complication of the right hand side terms increases with order. There is only a complementary solution for the zeroth-order governing equations. For orders greater than the zeroth, the solutions are composed of a complementary and a particular part. The complementary part is determined from the homogeneous part of that order's governing equations. The particular part is derived to satisfy the right hand side terms of the governing equations. The particular part is developed by using trial displacement functions with free constants, to match the right hand side terms of the governing equations of that order.

\section{B. The Coefficients for the First up to the Third-Order Solution}

The coefficients appearing in (21) are obtained as follows:

$$
\begin{aligned}
a_{2} & =\frac{1}{\widehat{\mu}}\left[3 A_{22}+\frac{v}{1-2 v}\left(4 A_{22}+2 C_{22}+\frac{1}{2} a_{1}\right)\right] \\
& \cdot\left[\frac{-2\left(\lambda^{10}-\lambda^{2}+1\right)}{\lambda^{2}\left(\lambda^{4}-1\right)\left(\lambda^{4}-\lambda^{2}+4\right)}\right],
\end{aligned}
$$




$$
\begin{array}{rlrl}
b_{2} & =\frac{1}{6 \widehat{\mu}}\left[3 A_{22}+\frac{v}{1-2 v}\left(4 A_{22}+2 C_{22}+\frac{1}{2} a_{1}\right)\right] & b_{3}=c_{3}-2 d_{3}-\frac{1}{8}\left(H_{2}(1)+H_{1}(1)\right), \\
\cdot\left[\frac{-2 \lambda^{10}-\lambda^{8}+2 \lambda^{6}+2 \lambda^{4}-3}{\lambda^{2}\left(\lambda^{4}-1\right)\left(\lambda^{4}-\lambda^{2}+4\right)}\right] & c_{3}=\frac{X_{3} X_{5}-X_{6} X_{2}}{X_{1} X_{5}-X_{4} X_{2}} \\
& -\frac{1}{6 \widehat{\mu}}\left[C_{22}-A_{22}\right], & d_{3}=\frac{X_{6} X_{1}-X_{3} X_{4}}{X_{1} X_{5}-X_{4} X_{2}} \\
c_{2}=\frac{1}{3 \widehat{\mu}}\left[3 A_{22}+\frac{v}{1-2 v}\left(4 A_{22}+2 C_{22}+\frac{1}{2} a_{1}\right)\right] & e_{3}=\frac{(2 / 3)\left[H_{3}(\lambda)-\lambda^{2} H_{3}(1)\right]}{\lambda^{2}-\lambda^{-4}}, \\
& \left.\cdot 1+\frac{2\left(1-\lambda^{2}-\lambda^{4}\right)}{\left(\lambda^{4}-1\right)\left(\lambda^{4}-\lambda^{2}+4\right)}\right], & f_{3}=\frac{(2 / 3)\left[H_{3}(\lambda)-\lambda^{-4} H_{3}(1)\right]}{\lambda^{2}-\lambda^{-4}}, \\
d_{2}=\frac{1}{2 \widehat{\mu}}\left[3 A_{22}+\frac{v}{1-2 v}\left(4 A_{22}+2 C_{22}+\frac{1}{2} a_{1}\right)\right] &
\end{array}
$$$$
\left[\frac{\lambda^{6}-1}{\lambda^{2}\left(\lambda^{4}-\lambda^{2}+4\right)}\right]
$$$$
e_{2}=\frac{1}{2 \widehat{\mu}}\left[\left(\frac{4 v^{2}-13 v+4}{2-4 v}\right) a_{1}+3 D_{22}\right] \frac{1-\lambda}{1-\lambda^{-4}},
$$$$
f_{2}=\frac{1}{2 \widehat{\mu}}\left[\left(\frac{4 v^{2}-13 v+4}{2-4 v}\right) a_{1}+3 D_{22}\right] \frac{\lambda\left(\lambda^{-5}-1\right)}{1-\lambda^{-4}},
$$

where

$$
\begin{aligned}
A_{11} & =\frac{-1}{1-2 v}\left[A_{1}(3-2 v)+\frac{1}{\widehat{\mu}} a_{1}(6-8 v)\right], \\
B_{11} & =\frac{2 v-3}{1-2 v} B_{1} \\
C_{11} & =\frac{1}{1-2 v}\left[\frac{1}{2} A_{1}(3-2 v)+\frac{1}{2} A_{1}(1+2 v)\right. \\
+ & \left.\frac{1}{2 \widehat{\mu}} a_{1}(6-8 v)\right], \\
D_{11} & =\frac{1}{1-2 v}\left[\left(3 A_{1}+B_{1}\right)-\frac{1}{\widehat{\mu}} a_{1}(6-8 v)\right], \\
A_{22} & =\frac{1}{2} \frac{1-2 v}{6-10 v}\left[B_{11}-A_{11}\right]-\frac{1}{2} \frac{1-2 v}{2-2 v}\left[A_{11}+B_{11}\right. \\
+ & \left.4 A_{1}\right] \\
C_{22} & =-C_{11} \frac{1-2 v}{9-10 v}, \\
D_{22} & =-D_{11} \cdot
\end{aligned}
$$

where

$$
\begin{aligned}
X_{1} & =8 \lambda-4 \lambda^{-3}-4 \lambda^{-5}, \\
X_{2} & =-18 \lambda+8 \lambda^{3}, \\
X_{3} & =-H_{1}(\lambda)+\frac{1}{2}\left[H_{2}(1)+2 H_{1}(1)\right] \lambda-\frac{1}{2}\left[H_{2}(1)\right. \\
& \left.+H_{1}(1)\right] \lambda^{3}, \\
X_{4} & =-8 \lambda+12 \lambda^{3}-4 \lambda^{-5}, \\
X_{6} & =18 \lambda-24 \lambda^{3}+6 \lambda^{-3}, \\
X_{6} & =-H_{2}(\lambda)-\frac{1}{2}\left[H_{2}(1)+2 H_{1}(1)\right] \lambda+\frac{3}{2}\left[H_{2}(1)\right. \\
& \left.+H_{1}(1)\right] \lambda^{3}, \\
& -\frac{1}{4}\left[e_{2} \varsigma^{-2}+f_{2} \varsigma^{2}+D_{22} \varsigma^{3}\right]+\frac{1}{4} A_{1} \varsigma^{3}-\frac{1}{2}\left[a_{2} \varsigma\right. \\
H_{1} & (\varsigma)=\left[\frac{4 L_{21}-3 v\left(L_{21}-L_{24}\right)}{1-2 v} \varsigma^{3}\right. \\
& \left.+\frac{1}{\widehat{\mu}} a_{1} \varsigma^{3}\right], \\
& +\frac{3 v\left(L_{22}+L_{25}\right)-2 L_{22}}{1-2 v} \varsigma^{-3} \\
& \left.+\frac{v\left(L_{23}+3 L_{26}\right)}{1-2 v} \varsigma^{-1}\right]+\left[b_{26}(3-4 v) \varsigma^{3}+c_{2} \varsigma^{-3} \varsigma^{3}+\frac{1}{2} C_{22} \varsigma^{3}+\frac{1}{4}\left(B_{1}-A_{1}\right) \varsigma^{3}\right.
\end{aligned}
$$

\section{The Coefficients for the Fourth-Order Solution}

The coefficients appearing in (22) are obtained as follows:

$$
a_{3}=4 c_{3}-9 d_{3}-\frac{1}{4}\left(H_{2}(1)+2 H_{1}(1)\right) \text {, }
$$




$$
\begin{aligned}
& \left.+b_{2}(-4 v) \varsigma^{3}-c_{2} \varsigma^{-3}+d_{2}(4 v-4) \varsigma^{-1}+A_{22} \varsigma^{3}\right] \\
& +\frac{1}{4 \widehat{\mu}} a_{1} \varsigma^{3} .
\end{aligned}
$$

In addition,

$$
\begin{aligned}
& L_{5}=\frac{1}{\widehat{\mu}} \frac{-1}{1-2 v}\left[3 b_{2}\left(8 v^{2}-6 v+1\right)+\frac{1}{2} A_{22}(1-2 v)\right. \\
& -C_{22}(1+v)+\frac{3}{4} B_{1}+A_{1}\left(7 v-\frac{23}{4}\right) \\
& \left.-\frac{9}{4} a_{1}(3-4 v)\right] \\
& L_{6}=-d_{2} \text {, } \\
& L_{7}=\frac{1}{\widehat{\mu}} \frac{1}{1-2 v}\left[\frac{-3}{4} a_{1}(3-4 v)+B_{1}(4 v-1)\right. \\
& +A_{1}(4 v-5)-6 b_{2}(v-1)+C_{22}(v+1) \\
& \left.+A_{22}(v-1)\right] \text {, } \\
& L_{8}=\frac{1}{\widehat{\mu}} \frac{1}{1-2 v}\left[6 c_{2}(1-v)\right] \text {, } \\
& L_{9}=\frac{1}{\widehat{\mu}} \frac{-1}{1-2 v}\left[d_{2}\left(-8 v^{2}+6 v+2\right)\right] \text {, } \\
& L_{10}=\frac{1}{\widehat{\mu}} \frac{-1}{1-2 v}\left[2 a_{2}(1-v)\right] \text {, } \\
& L_{11}=\frac{1}{\widehat{\mu}} \frac{1}{1-2 v}\left[\frac{-3}{4} a_{1}(3-4 v)+\frac{1}{4} B_{1}(16 v-5)\right. \\
& +\frac{1}{4} A_{1}(4 v-5)-2 b_{2}\left(10 v^{2}-20 v+9\right) \\
& \left.-C_{22}(3-5 v)-A_{22} v+D_{22}\right] \text {, } \\
& L_{12}=\frac{1}{\widehat{\mu}} \frac{-1}{1-2 v}\left[2 d_{2}(1-v)\right] \text {, } \\
& L_{13}=\frac{1}{1-2 v} e_{2} \\
& L_{14}=\frac{1}{1-2 v} f_{2} \text {, } \\
& L_{15}=\frac{3}{2} e_{2} \\
& L_{16}=\frac{-1}{2} f_{2} \text {, } \\
& L_{17}=\frac{1}{\widehat{\mu}} \frac{1}{1-2 v}\left[-D_{22}(1-2 v)+\frac{3}{2} a_{1}(3-4 v)\right. \\
& -\frac{1}{2} A_{1}(3+4 v)-\frac{1}{2} B_{1}(5-4 v)+12 b_{2}(1-2 v)
\end{aligned}
$$

$$
\begin{aligned}
+ & \left.4 A_{22}+2 C_{22}\right], \\
L_{18} & =\frac{1}{\widehat{\mu}} \frac{1}{1-2 v}\left[4 d_{2}(1-2 v)\right], \\
L_{19} & =\frac{-3}{2} f_{2}, \\
L_{20} & =\frac{1}{\widehat{\mu}} \frac{1}{1-2 v}\left[-2 D_{2}(1-2 v)+\frac{3}{4} a_{1}(3-4 v)\right. \\
& \left.-\frac{1}{2} A_{1}(3+4 v)+\frac{1}{2} B_{1}(1-4 v)\right] .
\end{aligned}
$$

The coefficients appearing in (C.3) are given as

$$
\begin{aligned}
& L_{21}=\frac{-1}{20 v-11} L_{1}, \\
& L_{22}=\frac{1}{20 v-11} L_{2}, \\
& L_{23}=\frac{-1}{20 v-11} L_{3}, \\
& L_{24}=\frac{1}{20 v-19} L_{7}, \\
& L_{25}=\frac{1}{20 v-19} L_{8}, \\
& L_{26}=\frac{-1}{20 v-19} L_{9} .
\end{aligned}
$$

\section{Conflict of Interests}

The authors declare that there is no conflict of interests regarding the publication of this paper.

\section{References}

[1] A. M. Fennimore, T. D. Yuzvinsky, W.-Q. Han, M. S. Fuhrer, J. Cumings, and A. Zetti, "Rotational actuators based on carbon nanotubes," Nature, vol. 424, no. 6947, pp. 408-410, 2003.

[2] L. Dong, X. Tao, M. Hamdi et al., "Nanotube fluidic junctions: internanotube attogram mass transport through walls," Nano Letters, vol. 9, no. 1, pp. 210-214, 2009.

[3] W. Yang, P. Thordarson, J. J. Gooding, S. P. Ringer, and F. Braet, "Carbon nanotubes for biological and biomedical applications," Nanotechnology, vol. 18, no. 41, Article ID 412001, 2007.

[4] A. R. Harutyunyan, B. K. Pradhan, G. U. Sumanasekera, E. Y. Korobko, and A. A. Kuznetsov, "Carbon nanotubes for medical applications," European Cells and Materials, vol. 3, no. 2, pp. 84$87,2002$.

[5] K. Kempa, J. Rybczynski, Z. Huang et al., "Carbon nanotubes as optical antennae," Advanced Materials, vol. 19, no. 3, pp. 421426, 2007.

[6] A. K.-T. Lau and D. Hui, "The revolutionary creation of new advanced materials-carbon nanotube composites," Composites Part B:Engineering, vol. 33, no. 4, pp. 263-277, 2002.

[7] A. Karlsson, R. Karlsson, M. Karlsson et al., "Molecular engineering: networks of nanotubes and containers," Nature, vol. 409, no. 6817, pp. 150-152, 2001. 
[8] G. E. Begtrup, W. Gannett, T. D. Yuzvinsky, V. H. Crespi, and A. Zettl, "Nanoscale reversible mass transport for archival memory," Nano Letters, vol. 9, no. 5, pp. 1835-1839, 2009.

[9] M. Zhang and J. Li, "Carbon nanotube in different shapes," Materials Today, vol. 12, no. 6, pp. 12-18, 2009.

[10] E. W. Wong, P. E. Sheehan, and C. M. Lieber, "Nanobeam mechanics: elasticity, strength, and toughness of nanorods and nanotubes," Science, vol. 277, no. 5334, pp. 1971-1975, 1997.

[11] C. Q. Ru, "Elastic buckling of single-walled carbon nanotube ropes under high pressure," Physical Review B, vol. 62, no. 15, pp. 10405-10408, 2000.

[12] X. Wang and H. Cai, "Effects of initial stress on non-coaxial resonance of multi-wall carbon nanotubes," Acta Materialia, vol. 54, no. 8, pp. 2067-2074, 2006.

[13] J. N. Reddy and S. D. Pang, "Nonlocal continuum theories of beams for the analysis of carbon nanotubes," Journal of Applied Physics, vol. 103, no. 2, Article ID 023511, 2008.

[14] Q. Wang, V. K. Varadan, and S. T. Quek, "Small scale effect on elastic buckling of carbon nanotubes with nonlocal continuum models," Physics Letters, Section A: General, Atomic and Solid State Physics, vol. 357, no. 2, pp. 130-135, 2006.

[15] K. Amara, A. Tounsi, I. Mechab, and E. A. Adda-Bedia, "Nonlocal elasticity effect on column buckling of multiwalled carbon nanotubes under temperature field," Applied Mathematical Modelling, vol. 34, no. 12, pp. 3933-3942, 2010.

[16] Y. Zhang, G. Liu, and X. Han, “Transverse vibrations of doublewalled carbon nanotubes under compressive axial load," Physics Letters, Section A: General, Atomic and Solid State Physics, vol. 340, no. 1-4, pp. 258-266, 2005.

[17] A. Benzair, A. Tounsi, A. Besseghier, H. Heireche, N. Moulay, and L. Boumia, "The thermal effect on vibration of single-walled carbon nanotubes using nonlocal Timoshenko beam theory," Journal of Physics D: Applied Physics, vol. 41, no. 22, Article ID 225404, 2008.

[18] L. Wang and H. Hu, "Flexural wave propagation in single-walled carbon nanotubes," Physical Review B-Condensed Matter and Materials Physics, vol. 71, no. 19, Article ID 195412, 2005.

[19] Y.-G. Hu, K. M. Liew, and Q. Wang, "Nonlocal elastic beam models for flexural wave propagation in double-walled carbon nanotubes," Journal of Applied Physics, vol. 106, no. 4, Article ID 044301, 2009.

[20] T. Murmu and S. C. Pradhan, "Thermo-mechanical vibration of a single-walled carbon nanotube embedded in an elastic medium based on nonlocal elasticity theory," Computational Materials Science, vol. 46, no. 4, pp. 854-859, 2009.

[21] Y. Gafour, M. Zidour, A. Tounsi, H. Heireche, and A. Semmah, "Sound wave propagation in zigzag double-walled carbon nanotubes embedded in an elastic medium using nonlocal elasticity theory," Physica E, vol. 48, pp. 118-123, 2013.

[22] A. Besseghier, A. Tounsi, M. S. A. Houari, A. Benzair, L. Boumia, and H. Heireche, "Thermal effect on wave propagation in double-walled carbon nanotubes embedded in a polymer matrix using nonlocal elasticity," Physica E: Low-Dimensional Systems and Nanostructures, vol. 43, no. 7, pp. 1379-1386, 2011.

[23] H. Heireche, A. Tounsi, A. Benzair, M. Maachou, and E. A. Bedia, "Sound wave propagation in single-walled carbon nanotubes using nonlocal elasticity," Physica E, vol. 40, no. 8, pp. 2791-2799, 2008.

[24] T. Murmu and S. C. Pradhan, "Thermal effects on the stability of embedded carbon nanotubes," Computational Materials Science, vol. 47, no. 3, pp. 721-726, 2010.
[25] A. C. Eringen, "Nonlocal polar elastic continua," International Journal of Engineering Science, vol. 10, no. 1, pp. 1-16, 1972.

[26] A. C. Eringen, "On differential equations of nonlocal elasticity and solutions of screw dislocation and surface waves," Journal of Applied Physics, vol. 54, no. 9, pp. 4703-4710, 1983.

[27] J. Peddieson, G. R. Buchanan, and R. P. McNitt, "Application of nonlocal continuum models to nanotechnology," International Journal of Engineering Science, vol. 41, no. 3-5, pp. 305-312, 2003.

[28] Y. Q. Zhang, G. R. Liu, and X. Y. Xie, "Free transverse vibrations of double-walled carbon nanotubes using a theory of nonlocal elasticity," Physical Review B, vol. 71, no. 19, Article ID 195404, 2005.

[29] Q. Wang, "Wave propagation in carbon nanotubes via nonlocal continuum mechanics," Journal of Applied Physics, vol. 98, no. 12, Article ID 124301, 2005.

[30] C. M. Wang, Y. Y. Zhang, S. S. Ramesh, and S. Kitipornchai, "Buckling analysis of micro- and nano-rods/tubes based on nonlocal Timoshenko beam theory," Journal of Physics D: Applied Physics, vol. 39, no. 17, article 3904, 2006.

[31] P. Lu, H. P. Lee, C. Lu, and P. Q. Zhang, "Dynamic properties of flexural beams using a nonlocal elasticity model," Journal of Applied Physics, vol. 99, no. 7, Article ID 073510, 2006.

[32] G. Domínguez-Rodríguez, A. Tapia, and F. Avilés, "An assessment of finite element analysis to predict the elastic modulus and Poisson's ratio of single-wall carbon nanotubes," Computational Materials Science, vol. 82, pp. 257-263, 2014.

[33] X. Wang and H. K. Yang, "Bending stability of multiwalled carbon nanotubes," Physical Review B, vol. 73, no. 8, Article ID 085409, 2006.

[34] D. Redekop, "A displacement solution in toroidal elasticity," International Journal of Pressure Vessels and Piping, vol. 51, no. 2, pp. 189-209, 1992. 

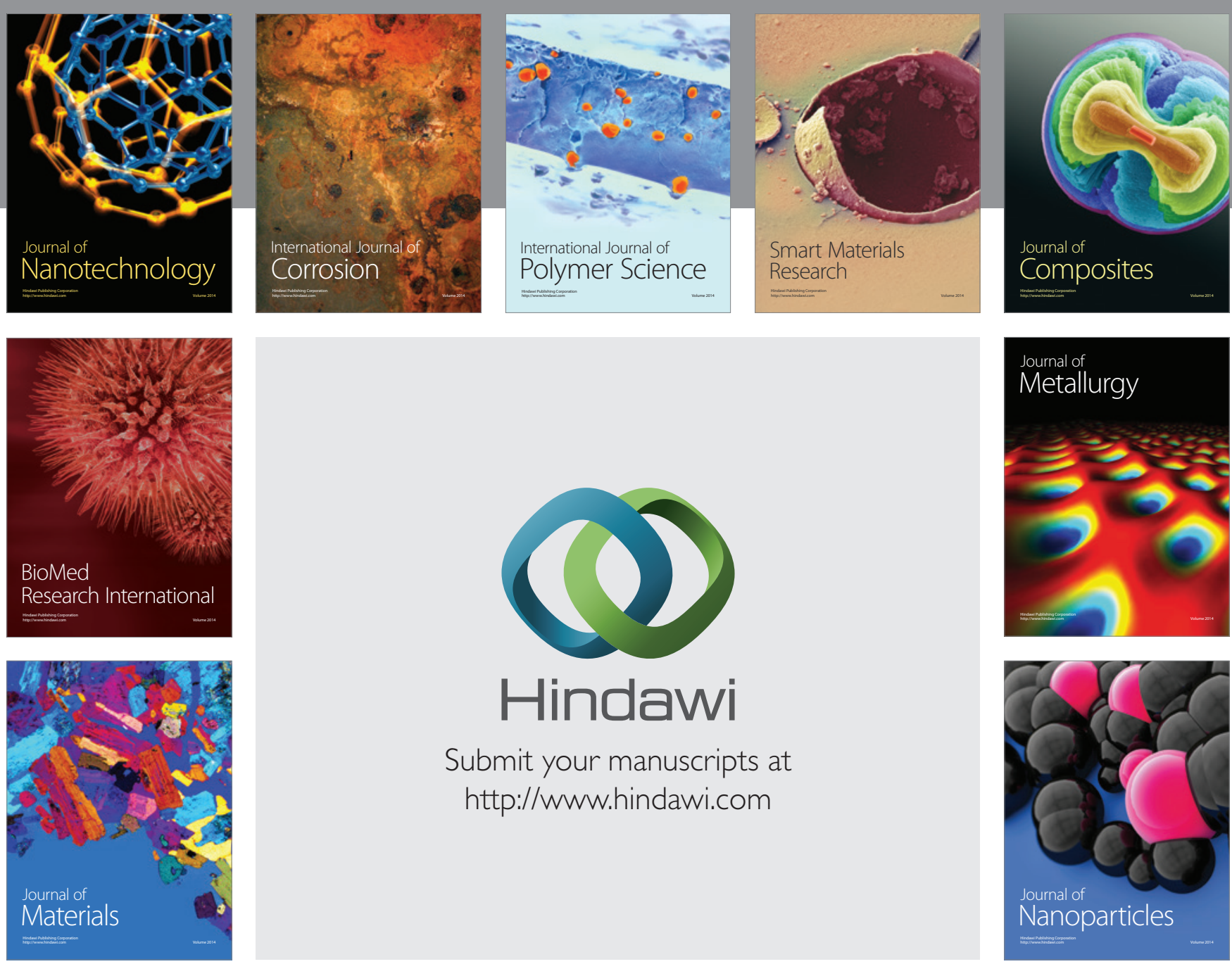

Submit your manuscripts at http://www.hindawi.com
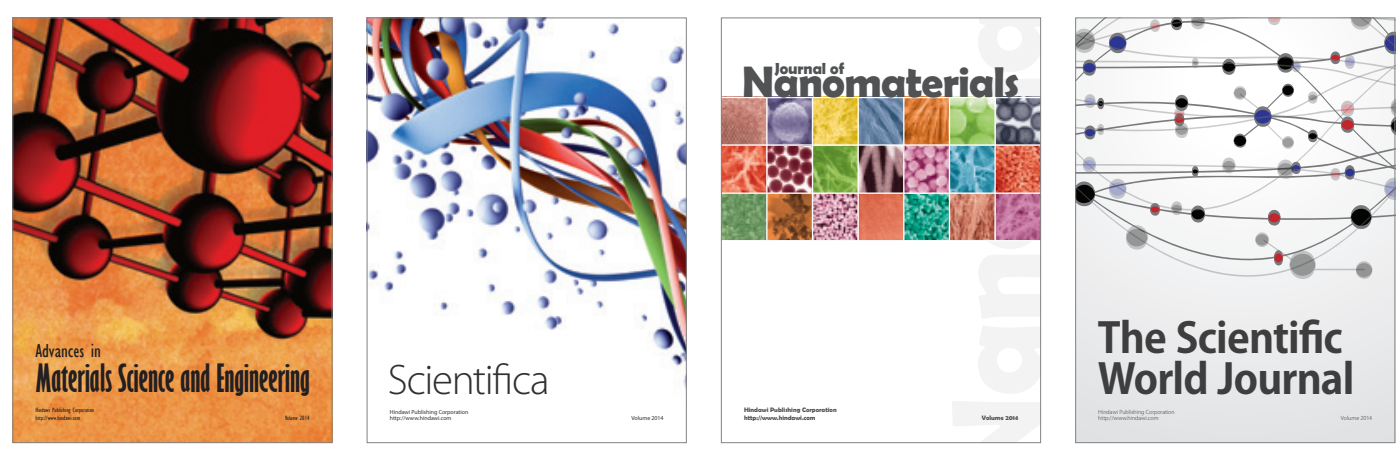

\section{The Scientific World Journal}
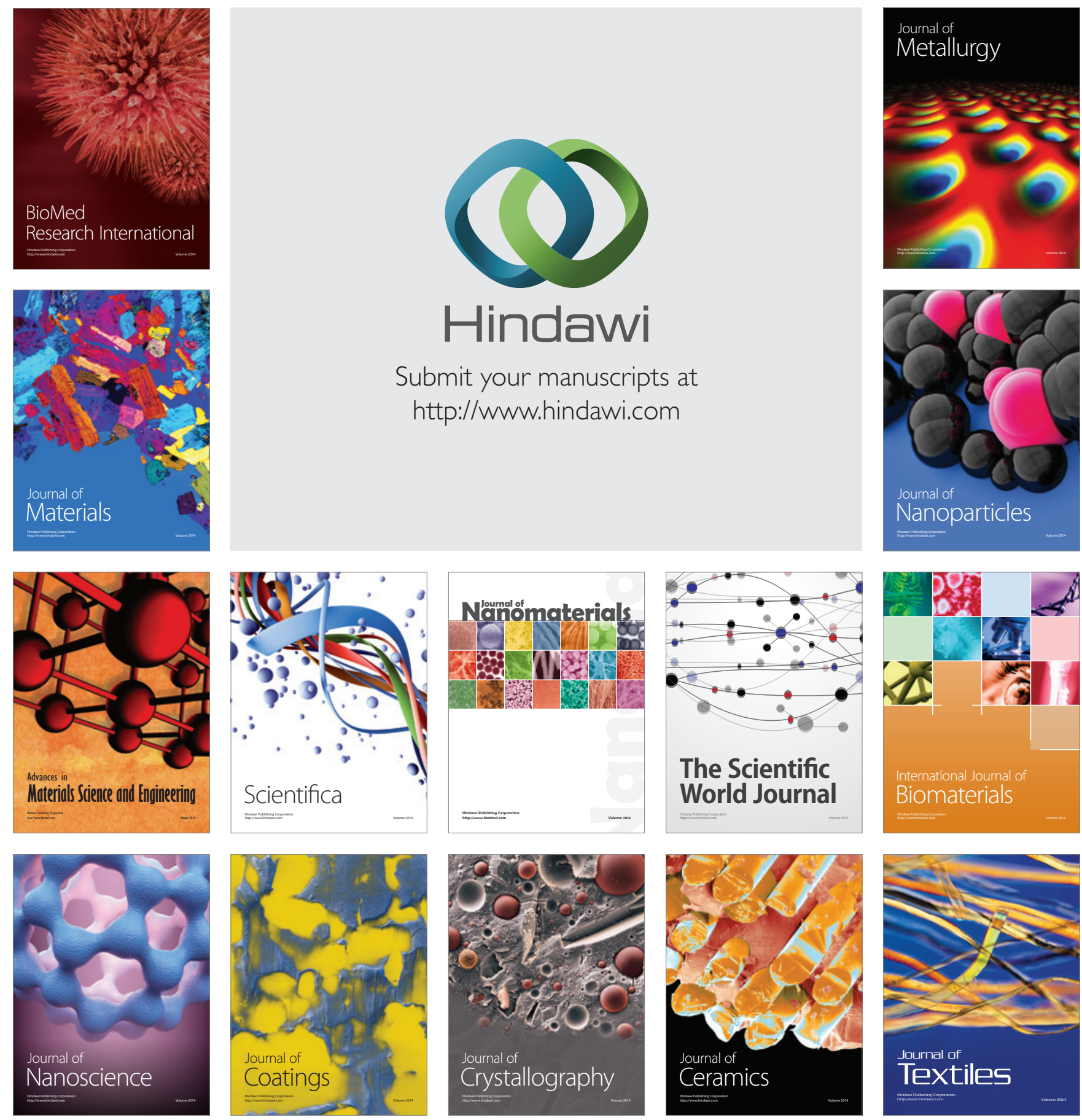